\title{
Caminhos (e descaminhos) dos objetivos em dissertações e teses: um olhar voltado para a coerência metodológica
}

\section{Leads and misleadings of the objectives in dissertations and theses: a look towards methodological coherence}

\author{
Marco Antonio F. da Costa ${ }^{1}$ \\ Maria de Fátima Barrozo da Costa \\ Viviane Abreu de Andrade $^{3}$
}

\section{RESUMO}

O estudo, bibliográfico e meta-analítico, foi realizado entre junho de 2012 e junho de 2013, em 197 monografias de programas da área de Educação e Ensino de Ciências, defendidas entre 2009 e 2012. Dessas, 92 eram dissertações de Mestrado Acadêmico (MA), 42 de Mestrado Profissional (MP) e 63 teses de Doutorado (DO). As monografias foram selecionadas em bancos de dados acadêmicos de instituições públicas e privadas brasileiras, disponibilizados na internet. A investigação teve como propósito verificar de que forma o elemento metodológico "objetivos" aparece nas dissertações e teses pesquisadas. Foram analisadas, também, a questão do gênero nas autorias das monografias e número de páginas. Como base de sustentação analítica, adotamos as características básicas da Metodologia da Pesquisa e a Taxonomia de Bloom. Os resultados apontaram para várias incoerências epistemológicas, como uso incoerente de verbos na formulação dos objetivos, e distribuição inadequada desse elemento metodológico nas estruturas das monografias.

Palavras-chave: Metodologia da Pesquisa; Objetivos de Investigação; Produção Acadêmica.

\section{ABSTRACT}

The bibliographic and meta-analytical research, was carried out between june 2012 and june 2013, analizing 197 monographs in the area of Education and Science Teaching, defended between 2009 and 2012, including 92 monographs of Academic Master's (MA), 42 of Professional Master's (MP) and 63 of Doctorate (DO). The dissertations and theses were selected in academic databases of brasilian public and private institutions, available on the internet. The research had the goal to verify in what form the methodological element "objectives" appeared in dissertations and theses. Were also analyzed, the matter of gender in authorship of the monographs and number of pages. We worked with the basic features of Research Methodology and Bloom's Taxonomy as analytical parameters. The results pointed to several epistemological inconsistencies, as mistaken use of verbs in the formulation of objectives, and inadequate distribution of this methodological element in the structures of monographs.

Keywords: Research Methodology; Research Objectives; Academic Production.

\footnotetext{
1 Professor da Escola Politécnica de Saúde Joaquim Venâncio (FIOCRUZ) e do Programa de Pós-Graduação em Ensino em Biociências e Saúde/ IOC - FIOCRUZ;

2 Pesquisadora da Escola Nacional de Saúde Pública Sérgio Arouca (ENSP);

3 Professora do Centro Federal de Educação Tecnológica Celso Suckow da Fonseca (CEFET/RJ), e doutoranda do Programa de Pós-Graduação em Ensino em Biociências e Saúde/IOC-Fiocruz-RJ.
} 


\section{INTRODUÇÃO}

Ao planejar uma pesquisa, na fase de projeto, é muito importante atentar para a linguagem textual utilizada em suas proposições, pois, a execução desse projeto levará a elaboração de uma monografia, que circulará no meio acadêmico, sendo, portanto, criticada e usada como referência em outras pesquisas. Dessa forma, há a necessidade de que o texto seja compreensível e preciso como demanda a escrita científica, e nessa linha, a linguagem dos elementos que compõem a monografia deve ser coerente, independente da natureza do trabalho científico, seja de cursos de Ensino Médio, graduação, especialização, mestrado (Dissertação) ou doutorado (Tese).

Neste estudo abordamos apenas dissertações e teses, e tratamos do elemento metodológico “objetivos”, o geral e os específicos, e outros elementos que, de alguma forma, tenham vínculo com os objetivos. A definição dos objetivos de uma dissertação ou tese é uma das bases de sustentação do processo de construção do conhecimento. Podemos, a partir deles, identificar a linha paradigmática do estudo, se a abordagem foi qualitativa ou quantitativa ou mista. A escolha do referencial teórico, ou mesmo da revisão de literatura, assim como do próprio caminho metodológico adotado, também estão diretamente vinculados aos objetivos.

Larocca et al. (2005: p.119) em estudo sobre a formulação de objetivos na pós-graduação em educação, aponta que "no contexto da pós-graduação, a preocupação em avaliar e comunicar adequadamente a produção acadêmica, certamente justifica-se mediante o reconhecimento de que a pesquisa deve gerar produtos fidedignos [...]."

André (2001: p. 63) ao escrever sobre a melhoria das condições dos trabalhos científicos na área de educação, diz que devemos assumir "como tarefa coletiva, o estabelecimento de critérios para avaliar as pesquisas da área [...]. Temos que continuar defendendo a qualidade nos trabalhos científicos e a busca do rigor."

Tomando por base essas considerações, buscamos, no estudo, verificar de que forma o elemento metodológico “objetivos" aparece nas dissertações e teses pesquisadas. Mesmo não tendo vinculação com os propósitos do estudo, analisamos, também, a questão do gênero e número de páginas, o que, de alguma maneira, pode contribuir para outras pesquisas.

Não tivemos o propósito de comparar resultados entre as instituições. Em alguns momentos algumas relações aparecerão, mas não serão identificados os locais de origem. Na maioria das instituições de ensino, as monografias são estruturadas segundo regras das próprias instituições, e, portanto, podem variar entre elas, porém, o sequenciamento analítico, adotado neste artigo, está baseado na coerência conceitual de cada elemento metodológico, que independe de regras institucionais (COSTA e COSTA, 2014; 2009).

\section{OS OBJETIVOS DE UMA PESQUISA}

Os objetivos de uma pesquisa estão diretamente relacionados às questões que foram formuladas. Fiorentini e Lorenzato (2006: p.91) afirmam que "uma vez definida a questão/pergunta da investigação, os objetivos da pesquisa podem ser, então, formulados ou revistos, caso já tenham sido inicialmente estabelecidos”. Os objetivos em um projeto é o que confere coerência ao plano de ação. O objetivo geral ressalta aonde o estudo pretende chegar ou alcançar, e, os objetivos específicos são as etapas que devem ser cumpridas para que possamos atender ao objetivo geral. Os objetivos, quando quantificados, recebem o nome de metas (COSTA e COSTA, 2014)

Larocca et al. (2005) em um estudo meta-analítico em 45 dissertações de mestrado em Educação, classificaram em seis categorias os objetivos formulados pelos autores dessas monografias, como aponta Cury (2012, p.241): 


\begin{abstract}
Compreensivos, avaliativos, propositivos, descritivos, objetivos-meio e objetivos generalistas. Os objetivos compreensivos, segundo os autores, visam interpretar uma dada realidade. Os avaliativos caracterizam-se pelas finalidades valorativas. Os propositivos, como o nome sugere, são relacionados a propostas de ações, planos ou alternativas. Já os descritivos pretendem relatar experiências ou narrar fatos e não comportam julgamentos do que é descrito. Os objetivos-meio, que não são objetivos de pesquisa, propriamente ditos, mas que servem para sustentar a pesquisa, bem como os objetivos generalistas, que são excessivamente amplos e podem servir para várias situações, gerando indefinição quanto ao que o investigador pretende obter.
\end{abstract}

Em razão dessas características, os objetivos devem ser formulados com uma linguagem compreensiva e precisa, devem ter uma lógica com o problema da pesquisa e também, devem ser factíveis de serem alcançados ao longo da pesquisa, portanto, é importante que a seleção dos verbos seja a mais adequada possível.

Uma das estratégias para a obtenção dessa lógica e coerência é o uso da Taxonomia de Bloom, que é uma metodologia que permite uma redação clara de objetivos. Essa classificação (BLOOM e KRATHWOHL, 1956) surgiu durante o desenvolvimento do paradigma construtivista (LOUSADA, 2012) e, embora ela seja aplicada nos processos de ensino-aprendizagem, já que classifica níveis e formas de aquisição do conhecimento, nada impede o seu uso na formulação dos objetivos de uma dissertação ou tese.

Segundo a Taxonomia de Bloom, a aprendizagem ocorre em três domínios: cognitivo, psicomotor e afetivo. Cognitivo: Envolve a aquisição de um novo conhecimento, do desenvolvimento intelectual, de habilidade e de atitudes. Afetivo: Está relacionado a sentimentos e posturas. Psicomotor: Relacionado a habilidades físicas específicas. O domínio cognitivo, que possui seis níveis: conhecimento, compreensão, aplicação, análise, síntese, e avaliação, pode, a nosso ver, sustentar de forma adequada a elaboração dos objetivos de uma monografia, como aponta Costa e Costa (2014), ao relacionar alguns verbos característicos de cada nível:

Nível de Conhecimento: Baseado na memorização, no armazenamento de informações. Comporta vários graus de complexidade, desde uma simples informação isolada, como uma data, um nome, e até o conhecimento de uma teoria ou estrutura. O que se deseja é a lembrança ou a retenção da informação apropriada. Verbos que devem ser utilizados: Definir, identificar, nomear, repetir, inscrever, listar, apontar, descrever (e outros).

Nível de Compreensão: Baseado no entendimento. Inclui a translação (passagem de uma mensagem de uma linguagem para outra), a interpretação (envolve o entendimento de interpelação das partes ou estrutura da mensagem) e a extrapolação (envolve predição de consequências da mensagem). Verbos que devem ser utilizados: descrever, discutir, organizar, interpretar, definir, debater (e outros).

Nível de Aplicação: Envolve a utilização dos conteúdos dos níveis de conhecimento e compreensão. Refere-se à capacidade de utilizar um material (conteúdo) apreendido em situações novas e concretas. Verbos que devem ser utilizados: Aplicar, demonstrar, descrever (e outros).

Nível de Análise: Envolve o desdobramento do material em suas partes consecutivas, a percepção de suas inter-relações e os modos de organização. Verbos que devem ser utilizados: Analisar, comparar, investigar, descrever (e outros).

Nível de Síntese: Envolve a organização de conteúdos trabalhados nos níveis de conhecimento, compreensão, aplicação e análise. Capacidade de combinar as partes para formar um todo. Neste nível deseja-se a projeção e criação de um produto original, valendo-se dos assuntos abordados. Verbos que devem ser utilizados: Propor, explicar, planejar, demonstrar (e outros).

Nível de Avaliação: É o nível de maior complexidade, pois implica em atividades de julgamento, isto é: uso de critérios e de padrões que permitam apreciar o grau de precisão, efetividade, economia ou suficiência de pormenores. Neste nível, o sujeito apresenta seu ponto de vista, o seu julgamento particular sobre o assunto 
tratado. Capacidade de julgar o valor de um material (conteúdo) com um dado propósito. Verbos que devem ser utilizados: Julgar, apreciar, comparar, avaliar, validar, analisar, demonstrar (e outros).

Para uma melhor compreensão desses níveis na elaboração dos objetivos, apresentamos uma possível aplicação, inerente ao Mestrado Profissional:

Para se desenvolver um produto, no caso do Mestrado Profissional, é necessário antes: buscar informações (conhecimento), compreender e analisar o processo de construção (compreensão e análise), explicar e avaliar/validar o produto (síntese e avaliação). Na definição do objetivo geral deve ser colocado um verbo que expresse aonde se quer chegar, por exemplo, pode-se propor ou desenvolver alguma coisa (nível de síntese), ou avaliar uma estratégia (nível de avaliação). Na elaboração dos objetivos específicos é muito importante levar esses níveis em consideração. Dessa forma, os verbos a serem utilizados para anunciar esses objetivos devem estar, segundo a Taxonomia de Bloom, em um nível de complexidade cognitiva, inferior ao nível do verbo utilizado na formulação do objetivo geral, já que os objetivos específicos são etapas para o atingimento do objetivo geral. Nos demais níveis monográficos, tal critério também deve ser levado em conta (COSTA e COSTA, 2014).

Em 2000, a Taxonomia de Bloom foi revisada (FERRAZ e BELHOT, 2010; ANDERSON e KRATHWOHL, 2001) e houve alteração nos nomes dos seis níveis, além de inverter as posições dos níveis de "síntese" (agora criar) e "avaliação" (agora avaliar). Como na taxonomia original, a versão revisada apresenta a classificação (Quadro 1) com alguns verbos que definem os objetivos:

Quadro 1 - Taxonomia de Bloom Revisada e alguns verbos correspondentes

\begin{tabular}{|l|l|}
\hline Níveis & Verbos \\
\hline Lembrar & Reconhecer, recordar, revisitar, descrever, definir \\
\hline Entender & Compreender, classificar, comparar, exemplificar, explicar, inferir, interpretar, resumir \\
\hline Aplicar & Realizar, executar, implementar, modificar \\
\hline Analisar & Atribuir, diferenciar, organizar, analisar \\
\hline Avaliar & Criticar, verificar, validar, avaliar \\
\hline Criar & Gerar, planejar, produzir, desenvolver, propor \\
\hline
\end{tabular}

A revisão da Taxonomia de Bloom original, de acordo com Anderson e Krathwohl (2001) buscou adaptá -la a uma perspectiva bidimensional. Diferentemente da versão de 1956, a taxonomia revisada diferencia "saber o quê" (o conteúdo do raciocínio expresso nos substantivos da classificação original - dimensão do conhecimento) de "saber como" (os procedimentos para resolver problemas expressos por verbos - dimensão dos aspectos e processos cognitivos). Embora tenha sofrido revisão, Fuller et al. (2007) apontam que a versão da taxonomia de Bloom mais utilizada ainda é a original.

\section{DESENHO METODOLÓGICO}

Bourdieu (1994, p. 23) cita que "a ciência é reforçada toda a vez que se reforça a crítica científica". Nessa linha, foi desenvolvida uma pesquisa bibliográfica (SÁ-SILVA et al., 2009), e meta-analítica, o que para Larocca et al. (2005: p.119) é onde:

[...]a ciência busca sua coerência, debruçando-se sobre aquilo mesmo que produz, não exclusivamente visando traçar o tradicional estado da arte de determinado conhecimento, mas para que, utilizando-se de procedimentos científicos qualitativos e/ou quantitativos, venha a conhecer melhor a produção científica em seus vários aspectos. 
O estudo foi realizado entre junho de 2012 e junho de 2013, em 17 bancos de dados de dissertações e teses defendidas entre 2009 e 2012, disponibilizados na internet pelas respectivas instituições públicas federais, estaduais, e particulares analisadas. Algumas instituições não possuíam dissertações e/ou teses em alguns desses anos, ou por não disponibilização na internet, ou por ausência de defesas. Para o atingimento dos propósitos do estudo, elencamos os seguintes fatores analíticos: gênero, número de páginas, abordagem paradigmática, resumos inadequados, presença do objetivo geral no resumo, presença de objetivos no sumário, resumo e introdução com verbos diferentes, presença de pergunta de pesquisa no corpo da monografia, presença de mais de uma pergunta de pesquisa, pergunta de pesquisa colocada no capítulo Procedimentos Metodológicos, pergunta de pesquisa depois da apresentação dos objetivos, objetivos iniciados sem verbo no infinitivo, objetivo geral com mais de um verbo, objetivo geral não declarado, presença de mais de três objetivos específicos, objetivos específicos não declarados, presença de objetivos formulados em linguagem da área de administração, objetivo geral iniciando a Introdução, objetivos no capítulo Procedimentos Metodológicos, objetivos em capítulo isolado, objetivos depois do capítulo Referencial Teórico, justificativa antes dos objetivos, e ausência de capítulo Procedimentos Metodológicos.

A Tabela 1 mostra, detalhadamente, a origem (por instituição), das 197 monografias selecionadas, sendo 131 oriundas de programas da área de Educação e 66 (itens 1 a 3, 11, 13, 14, 16, e 17) da área de Ensino de Ciências. Devido à recorrência dos dados, consideramos esse número total (197), como aceitável (CRESWELL, 2010). Foram 92 de Mestrado Acadêmico (MA), 42 de Mestrado Profissional (MP) e 63 de Doutorado (DO).

Tabela 1 - Instituições pesquisadas e respectivas monografias analisadas

\begin{tabular}{|c|c|c|c|c|c|}
\hline Ítem & Instituição & Sigla & MA & MP & DO \\
\hline 01 & $\begin{array}{l}\text { Centro Federal de Educação Tecnológica Celso } \\
\text { Suckow da Fonseca }\end{array}$ & CEFET/RJ & 4 & --- & --- \\
\hline 02 & $\begin{array}{l}\text { Instituto Federal de Educação, Ciência e Tecnologia } \\
\text { do Rio de Janeiro }\end{array}$ & IFRJ & --- & 8 & --- \\
\hline \multirow[t]{2}{*}{03} & Instituto Oswaldo Cruz / Fiocruz / RJ & IOC & 8 & 8 & 8 \\
\hline & Sub-Total & & 12 & 16 & 8 \\
\hline 04 & Universidade Federal Fluminense & UFF & 8 & --- & 7 \\
\hline 05 & Universidade Federal de Goiás & UFG & 8 & --- & 8 \\
\hline 06 & Universidade Federal de Minas Gerais & UFMG & 5 & --- & 6 \\
\hline 07 & Universidade Federal do Paraná & UFPA & 8 & --- & 8 \\
\hline \multirow[t]{2}{*}{08} & Universidade Federal do Rio de Janeiro & UFRJ & 8 & --- & 10 \\
\hline & Sub-Total & & 37 & --- & 39 \\
\hline 09 & Universidade de São Paulo & USP & 8 & --- & 8 \\
\hline 10 & Universidade Estadual de Londrina & UEL & 8 & --- & --- \\
\hline 11 & Universidade Estadual Paulista & UNESP & 8 & & 8 \\
\hline \multirow[t]{2}{*}{12} & Universidade Estadual do Rio de Janeiro & UERJ & 4 & --- & 4 \\
\hline & Sub-Total & & 28 & --- & 16 \\
\hline 13 & Centro Universitário UNIVATES / RS & UNIVATES & --- & 8 & --- \\
\hline 14 & Centro Universitário de Volta Redonda & UNIFOA & --- & 10 & --- \\
\hline 15 & Pontifícia Universidade Católica de Campinas & PUC-Campinas & 7 & --- & --- \\
\hline 16 & Universidade Estácio de Sá / RJ & UNESA & 8 & --- & --- \\
\hline \multirow[t]{3}{*}{17} & $\begin{array}{l}\text { Universidade do Grande Rio Prof. José de Sousa } \\
\text { Herdy }\end{array}$ & UNIGRANRIO & --- & 8 & --- \\
\hline & Sub-Total & & 15 & 26 & --- \\
\hline & TOTAL & & 92 & 42 & 63 \\
\hline
\end{tabular}

Fonte: Bancos de dados disponibilizados na internet pelas respectivas instituições. 
As instituições foram escolhidas em função da facilidade do acesso aos respectivos bancos de dados das produções acadêmicas. Não foram pesquisadas aquelas que exigiam para acesso, o conhecimento prévio da dissertação ou tese, como título, ou autor, e outras que não possuíam esse mecanismo de busca.

Essa facilidade no acesso foi devida a adesão dessas instituições a ideia de que o conhecimento precisa ser difundido de forma livre (CHALHUBI et al, 2012; SÁ, 2007; RODRIGUES, 2004) para que a sociedade possa se apropriar dele e, a internet tornou-se o principal vetor de distribuição de informações científicas. Pinheiro (2012, p.2) considera que:

[...] as bibliotecas digitais ou virtuais, aqui consideradas juntas, sem distinções conceituais que existem e são estudadas, estão entre serviços e produtos de informação contemporâneos. Essas bibliotecas reúnem documentos dispersos pelo mundo, além de trazer o seu texto completo, isto é, a informação sobre o documento e o próprio documento, e aquele que os buscou pode estar em qualquer lugar da Terra, não precisa se locomover. Na biblioteca física, convencional, o usuário terá que ir até o acervo, e na base de dados bibliográficos buscar as informações sobre o documento [...].

\section{RESULTADOS E DISCUSSÃO}

Após análise das 197 monografias, verificamos uma predominância do sexo feminino, conforme a Tabela 2:

Tabela 2 - Gênero nas monografias pesquisadas

\begin{tabular}{l|l|l|l|l|l|l}
\hline Gênero & \multicolumn{2}{|l|}{$\begin{array}{l}\text { Mestrado Acadêmico } \\
\text { MA (N=92) }\end{array}$} & \multicolumn{2}{l|}{$\begin{array}{l}\text { Mestrado Profissional } \\
\text { MP (N=42) }\end{array}$} & \multicolumn{2}{l}{$\begin{array}{l}\text { Doutorado } \\
\text { DO (N=63) }\end{array}$} \\
\hline \multirow{3}{*}{ Masculino } & $\mathrm{n}$ & $\%$ & $\mathrm{n}$ & $\%$ & $\mathrm{n}$ & $\%$ \\
Feminino & 20 & 22 & 15 & 36 & 23 & 37 \\
\hline
\end{tabular}

Fonte: Bancos de dados disponibilizados na internet pelas respectivas instituições.

Os valores dessa tabela estão de acordo com o perfil atual das Ciências Humanas e Sociais. A participação crescente de mulheres na pós-graduação já é um fato (SENKEVICS e POLIDORO, 2012; HAYASHI et al., 2007). Leta (2003) aponta que essa tendência foi observada a partir da segunda metade no século XX, em função até dos movimentos feministas, buscando a igualdade de direitos entre homens e mulheres.

Sobre o número de páginas das dissertações e teses, observamos, segundo a Tabela 3, uma variação bastante acentuada. Não temos, na literatura acadêmica uma definição de quantas páginas (mínimo e máximo) deve ter um texto monográfico. A nossa experiência docente e de orientação acadêmica mostra uma tendência acentuada para monografias mais objetivas, consequentemente com um número menor de páginas, embora, a coerência aponte para um número maior de páginas nos estudos de doutoramento, em função do seu grau analítico e detalhamento teórico-metodológico.

Tabela 3 - Número de páginas das monografias pesquisadas

\begin{tabular}{l|l|l|l}
\hline \multicolumn{1}{c|}{ Curso } & \multicolumn{1}{|c|}{$\begin{array}{c}\text { Número mínimo } \\
\text { de páginas }\end{array}$} & \multicolumn{1}{c|}{$\begin{array}{c}\text { Número médio de } \\
\text { páginas }\end{array}$} & $\begin{array}{c}\text { Número máximo } \\
\text { de páginas }\end{array}$ \\
\hline Mestrado Acadêmico & 45 & 131 & 331 \\
Mestrado Profissional & 74 & 121 & 210 \\
Doutorado & 88 & 219 & 469 \\
\hline
\end{tabular}

Fonte: Bancos de dados disponibilizados na internet pelas respectivas instituições. 
Uma característica que ainda demanda dúvidas por parte de alunos, e por que não dizer, também de alguns docentes, se refere à abordagem paradigmática do estudo, ou seja, se ela foi qualitativa (BAUER e GASKELL, 2011; FLICK, 2009), quantitativa (KULTAR, 2007) ou mista (CRESWELL, 2010). Na Tabela 4 apresentamos as abordagens encontradas no estudo.

Tabela 4 - Abordagens paradigmáticas nas monografias pesquisadas

\begin{tabular}{l|l|l|l|l|l|l|l}
\hline Abordagem & \multicolumn{2}{l|l}{$\begin{array}{l}\text { M e s t r a d o } \\
\text { Acadêmico } \\
\text { MA (N=92) }\end{array}$} & \multicolumn{2}{l|l}{$\begin{array}{l}\text { Mestrado Profissional } \\
\text { MP (N=42) }\end{array}$} & \multicolumn{2}{l}{$\begin{array}{l}\text { Doutorado } \\
\text { DO (N=63) }\end{array}$} \\
\hline \multirow{3}{*}{ Qualitativa } & $\mathrm{n}$ & $\%$ & $\mathrm{n}$ & $\%$ & $\mathrm{n}$ & $\%$ \\
Quantitativa & 84 & 92 & 30 & 72 & 60 & 95 \\
Mista (quali-quanti) & 03 & 03 & 01 & 02 & 02 & 03 \\
Não declarada & 02 & 02 & 01 & 02 & 01 & 02 \\
\hline
\end{tabular}

Fonte: Bancos de dados disponibilizados na internet pelas respectivas instituições.

Uma dissertação de MA, embora declarada quantitativa, apresentava como objetivo geral o verbo compreender, que é característico de abordagens qualitativas (que busca a compreensão de uma determinada realidade). Em contrapartida, em uma monografia de MP, com abordagem qualitativa, aparecia uma hipótese, elemento característico de abordagens quantitativas.

Em relação a não declaração do tipo de abordagem, principalmente nas 10 (24\%) dissertações de MP, consideramos um dado coerente com as nossas expectativas, já que o desenvolvimento de algum produto, processo ou estratégia, focos de um Mestrado Profissional, muitas vezes é estritamente técnico, não havendo, portanto, a necessidade de explicitar a abordagem analítica, se qualitativa, quantitativa ou mista, porém, no MA, é interessante que a abordagem seja explicitada. Nas três dissertações e uma tese, consideradas qualitativas-quantitativas (quali-quanti), ou seja, mistas, não observamos um desenho metodológico compatível com essa abordagem, isto é, não havia um desenho qualitativo, no sentido de compreender uma realidade, e também, posteriormente, um desenho quantitativo, para explicar essa realidade. Costa e Costa (2014: 38) acentuam que:

Atualmente temos verificado o uso cada vez maior da expressão “abordagem qualitativa-quantitativa”, simplesmente porque o estudo apresenta no seu contexto, números, percentuais, médias, histogramas, entre outros elementos. É importante frisar que a abordagem quantitativa possui vários requisitos (amostra representativa, hipótese a ser testada, um controle rigoroso de variáveis, entre outros). A abordagem qualitativa busca significados, não exige representatividade amostral, trabalha com pressupostos.

Os resultados pertinentes a vários fatores que permeiam, de alguma forma, direta ou indireta, o elemento metodológico “objetivos” (geral e específicos), encontram-se na Tabela 5. 
Tabela 5 - Características das monografias analisadas em relação a vários fatores

\begin{tabular}{|c|c|c|c|c|c|c|}
\hline \multirow[t]{2}{*}{ Parâmetros } & \multicolumn{2}{|c|}{$\begin{array}{l}\text { M e s t r a d o } \\
\text { Acadêmico } \\
\text { MA }(\mathrm{N}=92)\end{array}$} & \multicolumn{2}{|c|}{$\begin{array}{l}\text { M e s t r a d o } \\
\text { Profissional } \\
\text { MP }(\mathrm{N}=42)\end{array}$} & \multicolumn{2}{|c|}{$\begin{array}{l}\text { Doutorado } \\
\text { DO }(\mathrm{N}=63)\end{array}$} \\
\hline & $\mathrm{n}$ & $\%$ & $\mathrm{n}$ & $\%$ & $\mathrm{n}$ & $\%$ \\
\hline Resumos inadequados & 22 & 24 & 08 & 19 & 07 & 11 \\
\hline Presença de objetivo geral no Resumo & 33 & 36 & 08 & 19 & 15 & 24 \\
\hline Presença de objetivos (geral e específicos) no Sumário & 45 & 49 & 09 & 21 & 13 & 21 \\
\hline Resumo e Introdução com verbos diferentes nos objetivos & 06 & 07 & 04 & 10 & 02 & 03 \\
\hline Presença de pergunta de pesquisa no corpo da monografia & 75 & 86 & 29 & 69 & 48 & 76 \\
\hline Presença de mais de uma pergunta de pesquisa & 07 & 08 & 02 & 05 & -- & -- \\
\hline $\begin{array}{l}\text { Pergunta de pesquisa colocada no capítulo "Procedimentos } \\
\text { Metodológicos }\end{array}$ & 02 & 02 & -- & -- & -- & -- \\
\hline Pergunta de pesquisa depois da apresentação dos Objetivos & 12 & 13 & 13 & 31 & 05 & 08 \\
\hline Objetivos iniciados sem verbo no infinitivo & -- & -- & 01 & 02 & -- & -- \\
\hline Objetivo geral com mais de um verbo & 03 & 03 & 03 & 07 & 06 & 10 \\
\hline Objetivo geral não declarado & -- & -- & -- & -- & 06 & 10 \\
\hline Presença de mais de três objetivos específicos & 05 & 05 & 06 & 14 & -- & -- \\
\hline Objetivos específicos não declarados & 21 & 23 & 15 & 36 & 13 & 21 \\
\hline $\begin{array}{l}\text { Presença de objetivos formulados em linguagem da área de } \\
\text { administração }\end{array}$ & -- & -- & -- & -- & 01 & 02 \\
\hline Objetivo geral iniciando a Introdução & 03 & 03 & -- & -- & 01 & 02 \\
\hline Objetivos no capítulo "Procedimentos Metodológicos" & 03 & 03 & -- & -- & -- & -- \\
\hline Objetivos em capítulo isolado & 01 & 01 & -- & -- & -- & -- \\
\hline Objetivos depois do capítulo "Referencial Teórico” & 02 & 02 & 01 & 02 & -- & -- \\
\hline Justificativa antes dos objetivos & 03 & 03 & 01 & 02 & -- & -- \\
\hline Ausência de capítulo "Procedimentos Metodológicos" & -- & -- & -- & -- & 07 & 11 \\
\hline
\end{tabular}

Fonte: Bancos de dados disponibilizados na internet pelas respectivas instituições.

Os percentuais relativos à verificação de resumos incoerentes é um fator preocupante, haja vista, a importância desse elemento nos processos de busca em bancos de dados. Os resumos citados não apresentavam configuração adequada (SILVA e MATA, 2002), sendo, na realidade, uma ingênua introdução ao trabalho, sem indicação do problema, objetivos, justificativa, desenho metodológico, resultados e conclusões. Salomon (2008, p. 186-187) diz que um resumo é "apresentação concisa e frequentemente seletiva do texto de um artigo, obra ou outro documento, pondo em relevo os elementos de maior interesse e importância [...]”.

A baixa presença dos objetivos nos sumários de 45 (49\%) dissertações de MA, nove (21\%) do MP e13 (21\%) do DO, são dados que merecem atenção, já que esse elemento é, geralmente, àquele em que o leitor tem o primeiro contato com a monografia, e a sua ausência pode gerar descrédito, ou até mesmo falta de interesse em continuar a leitura.

Um dado que nos chamou a atenção foi à presença de verbos diferentes para o objetivo geral no resumo e na introdução, fato observado nas dissertações de MA e MP e nas teses. Encontramos em uma monografia de MA, no resumo o verbo analisar e na introdução o verbo compreender, em uma de MP, o verbo avaliar no resumo e discutir na introdução, e em uma tese, o verbo contextualizar no resumo e contribuir na introdução. A 
manutenção dos mesmos verbos, selecionados para os objetivos, ao longo de toda a monografia, é um cuidado que os alunos devem ter, para que não haja qualquer incompreensão sobre o texto.

Os resultados inerentes à presença de pergunta de pesquisa estão compatíveis com o esperado, inclusive para as 29 (69\%) monografias de MP, que tem como objetivo a geração de um produto, e nesse contexto a ausência de pergunta é aceitável, embora a sua presença seja sempre sugerida. Entretanto, deve-se ressaltar que, de acordo com as boas práticas metodológicas, a colocação de mais de uma pergunta de pesquisa, observada nas monografias do MA (três com cinco perguntas e uma com sete, as demais com quatro) e nas monografias do MP (uma com seis e outra com três questões), implica na elaboração de resposta para cada uma delas (COSTA e COSTA, 2014; CRESWELL, 2010).

A pergunta atua como um vetor, a fim de orientar o caminho a ser percorrido ao longo do estudo (MINAYO, 2010). Consideramos que o local mais adequado para a colocação da pergunta, é antes dos objetivos, porque a questão nada mais é do que a "síntese do problema de pesquisa”, e a partir daí, os objetivos, que representam o "para quê da pesquisa” devem aparecer. Em termos epistemológicos, tal sequência de elementos metodológicos no texto, fica mais coerente. A colocação de pergunta no capítulo "Desenho Metodológico" é totalmente incompatível, já que esse capítulo representa o “como” da pesquisa, e, portanto, epistemologicamente, não cabe a inserção de questionamentos nessa seção do texto monográfico.

Não é adequada a formulação de mais de três objetivos específicos, notado em cinco (5\%) das dissertações de MA, e seis (14\%) nas de MP, já que implica, necessariamente, em que todos eles sejam atingidos ao longo do estudo. Esse fato, geralmente, leva a um maior número de páginas, e muitas vezes, não agrega valor à monografia, portanto, é uma escolha que deve ser bem pensada. As cinco monografias de MA nas quais encontramos mais de três objetivos específicos, foram desenvolvidas em uma mesma instituição. Esse dado pode indicar uma possível diretriz metodológica recomendada pela própria instituição.

Um resultado surpreendente foi à ausência de objetivo geral em seis (10\%) teses de Doutorado, sendo que quatro delas eram da mesma instituição. Isso pode apontar para uma influência do processo de ensino da disciplina Metodologia da Pesquisa, falhas na orientação, ou até mesmo, de um padrão da instituição. Também no Doutorado, com o mesmo percentual (10\%), encontramos a presença de mais de um verbo no objetivo geral. Nesse nível de estudo, em que já se espera um amadurecimento metodológico, esses dados são preocupantes. O objetivo geral é único, assim como também, em cada objetivo específico deve haver apenas um verbo (FAGUNDES, 2008). Em duas monografias de MA, duas de MP, e uma de DO, encontramos objetivos com dois verbos. Em uma de DO, o objetivo geral foi definido com três verbos.

A não declaração de objetivos específicos, mostrados na Tabela 5, nas 21 (23\%) dissertações de MA, 15 (36\%) do MP, 21 (23\%) do MA, e 13 (21\%) do Doutorado, é uma falha metodológica grave, já que para se alcançar qualquer objetivo geral, há necessidade, antes, de se concretizar algumas ações intermediárias, que são os objetivos específicos.

Uma configuração de objetivos incomum nas áreas de Educação e de Ensino de Ciências foi encontrada em uma tese de Doutorado, que apresentava no objetivo geral o verbo analisar, e dividia os objetivos específicos em macro (com o verbo analisar), meso (com o verbo identificar), e micro (com os verbos caracterizar, identificar e contribuir). Esses prefixos gregos significam: macro=longo, meso=meio e micro=pequeno. $\mathrm{O}$ uso desses prefixos se aplica na área de Administração, principalmente em planejamento estratégico, no contexto dos objetivos organizacionais, não sendo, portanto, indicado para a formulação de objetivos em dissertações e teses em Ciências Humanas e Sociais, que não tem, nesse elemento metodológico, como princípio, a hierarquização das ações (FERREIRA, 2009).

O início da Introdução com o objetivo geral em três (3\%) dissertações do MA, e uma (2\%) do DO, e a presença dos objetivos (geral e específicos) no capítulo "Desenho Metodológico", encontrado em três (3\%) das dissertações de MA, também deve ser evitada, já que, como dito anteriormente, os objetivos devem estar após a 
pergunta de pesquisa (FIORENTINI e LORENZATO, 2006). No capítulo “Desenho Metodológico” utiliza-se de forma corrente, verbos no infinitivo para expressar procedimentos metodológicos, como, por exemplo: realizar oficinas, aplicar questionários, entre outros.

A apresentação de objetivos em capítulo próprio foi observada em apenas uma dissertação de MA. Tal colocação é uma característica da área de Ciências Exatas e Biomédicas. Na área de Ciências Humanas e Sociais é fato raríssimo, mas não há incoerência metodológica nessa configuração. Já, inserir os objetivos depois do capítulo "Referencial Teórico", como mostrado na Tabela 5, em duas (2\%) das monografias de MA e uma (2\%) do MP, é uma prática que deve ser evitada, pois a seleção de referenciais que irão suportar o desenvolvimento da pesquisa considera exatamente os objetivos do estudo. Desse modo, é necessário que os objetivos sejam colocados no corpo da monografia, antes do capítulo "Referencial Teórico”, para que a coerência metodológica seja mantida.

Outro dado não esperado, embora de baixa incidência, foi a presença da justificativa da pesquisa, antes dos objetivos, em três (3\%) de MA e uma (2\%) do MP. Esse elemento (justificativa) é o "por quê" do estudo, portanto, não é adequado colocá-lo antes dos objetivos "para quê". Em outras palavras, para se justificar uma pesquisa, é necessário, antes, apresentar os seus objetivos.

Em relação aos verbos usados para elaboração dos objetivos, o estudo identificou o uso de 71 verbos diferentes, dos quais 26 para o objetivo geral e os demais, 45, para os objetivos específicos. Para efeito de análise, consideramos apenas os cinco verbos mais citados, conforme as Tabelas 6, 7 e 8. Para fins de praticidade no entendimento, optamos por fazer a análise dessas tabelas em conjunto. No caso de verbos com o mesmo número de citações, selecionamos àqueles que consideramos mais apropriados para análise.

Tabela 6 - Os cinco verbos mais utilizados na elaboração do objetivo geral e específicos nas monografias de Mestrado Acadêmico pesquisadas ( $\mathrm{N}=92)$

\begin{tabular}{l|l|l|l|l|l}
\hline \multirow{2}{*}{ Verbos } & \multicolumn{2}{l|}{ Obj. Geral } & \multirow{2}{*}{ Verbos } & & \multicolumn{2}{l}{ Objs. Específicos } \\
\cline { 2 - 3 } & $\mathbf{n}$ & $\mathbf{0}$ & & $\mathbf{n}$ & \% \\
\hline Analisar & 21 & 23 & Analisar & 14 & 15 \\
Investigar & 15 & 16 & Identificar & 11 & 12 \\
Compreender & 14 & 15 & Investigar & 07 & 08 \\
Identificar & 5 & 05 & Descrever & 05 & 05 \\
Descrever & 5 & 05 & Contribuir & 04 & 04 \\
\hline
\end{tabular}

Fonte: Bancos de dados disponibilizados na internet pelas respectivas instituições.

Tabela 7 - Os cinco verbos mais utilizados na elaboração do objetivo geral e específicos nas monografias de Mestrado Profissional pesquisadas ( $\mathrm{N}=42$ )

\begin{tabular}{l|l|l|l|l|l}
\hline \multirow{2}{*}{ Verbos } & \multicolumn{2}{l|l}{ Obj. Geral } & \multirow{2}{*}{ Verbos } & \multicolumn{2}{l}{ Objs. Específicos } \\
\cline { 2 - 3 } & $\mathbf{n}$ & $\mathbf{0}$ & & $\mathbf{n}$ & \% \\
\hline Investigar & 08 & 19 & Demonstrar & 05 & 12 \\
Desenvolver & 06 & 14 & Analisar & 05 & 12 \\
Propor & 05 & 12 & Mapear & 03 & 07 \\
Avaliar & 04 & 10 & Implementar & 02 & 05 \\
Refletir & 02 & 05 & Produzir & 02 & 05 \\
\hline
\end{tabular}

Fonte: Bancos de dados disponibilizados na internet pelas respectivas instituições. 
Tabela 8 - Os cinco verbos mais utilizados na elaboração do objetivo geral e específicos nas monografias de Doutorado pesquisadas $(\mathrm{N}=63)$

\begin{tabular}{l|l|l|l|l|l}
\hline \multirow{2}{*}{ Verbos } & \multicolumn{2}{l}{ Obj. Geral } & \multirow{2}{*}{ Verbos } & \multicolumn{2}{l}{ Objs. Específicos } \\
\cline { 2 - 3 } \cline { 5 - 6 } & $\mathbf{n}$ & $\mathbf{0}$ & & $\mathbf{n}$ & $\mathbf{\%}$ \\
\hline Analisar & 12 & 19 & Contribuir & 08 & 13 \\
Compreender & 07 & 11 & Identificar & 07 & 11 \\
Investigar & 06 & 10 & Avaliar & 05 & 08 \\
Propor & 05 & 08 & Descrever & 05 & 08 \\
Revisitar & 02 & 03 & Refletir & 03 & 05 \\
\hline
\end{tabular}

Fonte: Bancos de dados disponibilizados na internet pelas respectivas instituições.

Notamos uma grande predominância dos verbos analisar e investigar, que podemos considerar sinônimos, nas dissertações e teses pesquisadas. Esses verbos, que na Taxonomia de Bloom estão no nível de análise, são aqueles que em função da nossa própria experiência docente em Metodologia da Pesquisa, também observamos nos processos monográficos, tanto no objetivo geral, como nos específicos. Realmente esses verbos expressam inter-relações, e identificam componentes críticos do estudo para análise, principalmente em estudos com abordagens qualitativas.

O verbo compreender, presente no objetivo geral de dissertações de MA e teses, está coerente com a linha paradigmática adotada na maioria dessas monografias (abordagem qualitativa, conforme a Tabela 4) e se encontra no nível de compreensão da Taxonomia de Bloom, principalmente porque os objetos de estudo exigem interpretação, fator característico das abordagens qualitativas (COSTA e COSTA, 2013).

Nas monografias de MP percebemos uma coerência metodológica e cognitiva em relação aos objetivos gerais e específicos e também, frente ao propósito de um mestrado profissional, que é a geração de um produto, ou seja, os verbos mais utilizados no objetivo geral, como: investigar, desenvolver, propor e avaliar, se encontram nos níveis de síntese e avaliação da Taxonomia de Bloom, e os específicos estão, cognitivamente alinhados.

O verbo refletir, também citado na Tabela 8 (objetivos específicos do DO), e o verbo contribuir, nos objetivos específicos do MA, são verbos que não devemos usar na formulação de objetivos, já que eles são intangíveis para o estudo, isto é, ao término da pesquisa não teremos condições de verificar se realmente houve reflexão ou alguma contribuição. Para isso, teríamos que desenvolver um novo estudo. Outros verbos nessa linha, como: motivar, fortalecer, conscientizar, entre outros, são úteis para a formulação do elemento metodológico "Resultados esperados” e/ou "Impactos do estudo”, nem sempre presentes em projetos de pesquisa de Mestrado e Doutorado, apenas naqueles enviados para agências de fomento, tais como: CNPq, Faperj, entre outros (COSTA e COSTA, 2014). Esses verbos não constam do domínio cognitivo na Taxonomia de Bloom.

Na pesquisa, de forma geral, nos deparamos com verbos “estranhos” a linguagem acadêmica, como: revisitar, no objetivo geral de uma tese de Doutorado, percorrer, confrontar, trazer, apurar, nos objetivos específicos em dissertações de MP, agregar, detectar, esgotar, instigar e deslocar, também nos objetivos específicos de algumas dissertações de MA. É importante que, na formulação de objetivos, o significado do verbo não seja ambíguo ou passível de diferentes interpretações. Embora todos os exemplos citados, estivessem contextualizados no nível de conhecimento da Taxonomia de Bloom, ou seja, na busca de informações, e na identificação de elementos específicos, os mesmos poderiam gerar dúvidas em relação ao atingimento dos objetivos propostos, o que não é compatível com a linguagem científica (COSTA e COSTA, 2014; CRESWELL, 2010; MINAYO, 2010). 


\section{CONSIDERAÇÕES FINAIS}

Quando os objetivos e outros elementos monográficos não estão claros ou se estão escritos de forma incoerente, perde-se muito daquilo que chamamos de "qualidade epistemológica”, ou seja, embora a produção do conhecimento ocorra, ela poderá ter sido gerada com uma linguagem inadequada, e isso poderá fazer com que outros pesquisadores tenham dificuldades em realizar pesquisas semelhantes, e em alguns casos, até colocar em dúvida a pertinência do estudo e da própria instituição de origem.

O fato de que as dissertações e teses passam por várias instâncias de avaliação, como: seminários de avaliação de resultados, qualificações, defesa, e as orientações ao longo do processo, e comparando, qualitativamente, com os resultados deste estudo, nos perguntamos: Por quê as inadequações estruturais e metodológicas encontradas não foram identificadas a tempo de serem corrigidas antes das respectivas defesas e/ou divulgação do trabalho? A resposta para essa pergunta, acreditamos, deve ser buscada em pesquisas posteriores, por pesquisadores que transitam no campo da Metodologia da Pesquisa.

Portanto, esse estudo revelou, implicitamente, a importância que deve ser dada a disciplina Metodologia da Pesquisa e a formação dos seus professores, que entendemos, devem estar familiarizados com os processos de pesquisa, e que não conheçam esse campo apenas por livros.

É importante realçar esta última afirmação, pois há uma vasta produção e comercialização de livros sobre Metodologia da Pesquisa, com diferentes configurações metodológicas, e muitos sem uma discussão epistemológica sobre os elementos que compõe um processo monográfico, tratando, na maioria das vezes, apenas de mecanismos de referenciação bibliográfica. Esse fato pode, a nosso ver, exercer influência na compreensão e na elaboração inadequada de alguns elementos metodológicos, como no caso dessa pesquisa, o elemento "objetivos”. Verificamos, também, no percurso de construção desse estudo, que ainda existe pouca produção acadêmica nos periódicos nacionais, relacionada a artigos científicos envolvendo a Metodologia da Pesquisa.

Ademais, acreditamos que os processos de ensino praticados na docência da Metodologia da Pesquisa devem ser pautados por uma discussão conceitual abrangente dos elementos pertinentes a uma monografia, mostrando claramente os seus limites epistemológicos, para que tenhamos dissertações e teses adequadamente construídas, e dessa forma, garantindo o rigor científico, e facilitando o pensar correto dos alunos, possamos avançar na construção e na busca do conhecimento.

\section{REFERÊNCIAS}

ANDERSON, L. W.; KRATHWOHL, D. R.A taxonomy for learning, teaching, and assessing. New York: Longman, 2001.

ANDRÉ, M. Pesquisa em Educação: buscando rigor e qualidade. Cadernos de Pesquisa, São Paulo, n. 113, 2001, pp. 51-64.

BAUER, M.W.; GASKELL, G.F. Pesquisa Qualitativa com Texto, Imagem e Som. 9 Edição. Petrópolis: Ed. Vozes, 2011.

BLOOM, B. S.; KRATHWOHL, D. R. Taxonomy of Educational Objectives. Handbook I: Cognitive Domain. New York: Longmans Green and Company, 1956.

BOURDIEU, P. Lições da aula. 2. Edição. São Paulo: Ed. Ática, 1994.

CHALHUBI, T; BENCHIMOL, A; GUERRA, C. III Encontro de Biblioteconomia. Revista Eletrônica de Biblioteconomia e Ciência da Informação, UFSC, v. 17, n. esp. 2 - III SBCC, 2012, p.p. 159-173. 
COSTA, M.A.F.; COSTA, M.F.B. Projeto de Pesquisa: entenda e faça. 5. Edição. Rio de Janeiro: Ed. Vozes, 2014.

COSTA, M.A.F.; COSTA, M.F.B. Metodologia da Pesquisa: conceitos e técnicas. 2. Edição. Rio de Janeiro: Ed. Interciência, 2009.

CRESWELL, J.W. Projeto de Pesquisa: métodos qualitativo, quantitativo e misto. 3. Edição. Porto Alegre: Ed. Artmed, 2010.

CURY, H.N. Pesquisas em ensino de ciências e matemática, relacionadas com erros: uma investigação sobre seus objetivos. Revista Educação Matemática Pesquisa, São Paulo, v.14, n.2, 2012, pp.237-256.

FAGUNDES, A.J.F.M. Cuidados para a formulação de objetivos de pesquisa. Revista Educação, São Paulo, v.3, n.1, 2008, pp. 73-81.

FERRAZ, A. P. C. M.; BELHOT, R. V. Taxonomia de Bloom: Revisão teórica e apresentação das adequações do instrumento para definição de objetivos instrucionais. Gestão \& Produção, n.17, v.2, 2010, pp. 421-431.

FERREIRA, R.R. Avaliação de necessidades de treinamento: proposição e aplicação de um modelo teóricometodológico nos níveis macro e meso organizacionais. 2009. 219 f. Dissertação (Mestrado em Psicologia Social, do Trabalho e das Organizações)-Universidade de Brasília, Brasília, 2009.

FIORENTINI, D.; LORENZATO, S. Investigação em Educação Matemática: percursos teóricos e metodológicos. Campinas: Autores Associados, 2006.

FLICK, U. (coord.). Coleção Pesquisa Qualitativa. Porto Alegre: Ed. Bookman, 2009.

FULLER, U.; JOHNSON, C.G.; AHONIEMI, T.; et. al. Developing a Computer Science-Specific Learning Taxonomy. SIGCSE Bulletin, USA, v. 39, n. 4, 2007, pp. 152-170.

KULTAR, S. Quantitative Social Research Methods. Sage: Thousand Oaks, CA/ USA, 2007.

HAYASHI, M.C.P.I.; CABRERO, R.C.; COSTA, M.P.R.; HAYASHI, C.R.M. Indicadores da participação feminina em Ciência e Tecnologia. TransInformação, PUC-Campinas, v.19, n.2, 2007, pp. 169-187.

LAROCCA, P.; ROSSO, A.J.; SOUZA, A.P. A formulação dos objetivos de pesquisa na pós-graduação em Educação: uma discussão necessária. RBPG, v.2, n.3, 2005, pp. 118-133.

LETA, J. As mulheres na ciência brasileira: crescimento, contrastes e um perfil de sucesso.Estudos Avançados, v.17, n.49, 2003, pp. 271-284.

LOUSADA, I.H. Diseño de software educativo para la enseñanza de la programación orientada a objetos basado en la taxonomía de Bloom. Tesis Doctoral. Universidad Rey Juan Carlos - Escuela Técnica Superior de Ingeniería Informática. España, 2012.

MINAYO, M.C.S. (org.). Pesquisa social: teoria, método e criatividade. 29. Edição. Petrópolis: Ed. Vozes, 2010. (Coleção temas sociais).

PINHEIRO, L.V.R. Internet, Ciência e Sociedade: o que mudou para pesquisadores. ComCiência: Revista Eletrônica de Jornalismo Científico, n.139, 2012, pp.1-3.

RODRIGUES, E. Acesso livre ao conhecimento: a mudança do sistema de comunicação da ciência e os profissionais de informação. Cadernos BAD, vol. 2004, n. 1, 2004, pp. 24-35.

SÁ, A.V.M. Acesso livre eletrônico: mudança paradigmática na difusão do conhecimento científico em educação. Linhas Críticas, UnB, vol. 13, n. 24, 2007, pp. 3-4. 
SÁ-SILVA, J.R.; ALMEIDA, C.D.; GUINDANI, J.F. Pesquisa documental: pistas teóricas e metodológicas. Revista Brasileira de História \& Ciências Sociais, ano1, n.1, 2009, pp. 1-15.

SALOMON, D. V. Como fazer uma monografia. 11. Edição. São Paulo: Martins Fontes, 2008.

SENKEVICS, A.S.; POLIDORO, J.Z. Corpo, gênero e ciência: na interface entre biologia e sociedade. Revista da Biologia, USP, v.9, n.1, 2012, pp.16-21.

SILVA, J.Q.G.; MATA, M.A. Proposta tipológica de resumos: um estudo exploratório das práticas de ensino da leitura e da produção de textos acadêmicos. SCRIPTA, Belo Horizonte, v. 6, n. 11, 2002, pp. 123-133. 